Neurocase. 2018 April ; 24(2): 90-94. doi:10.1080/13554794.2018.1447134.

\title{
A case of semantic variant primary progressive aphasia with Pick's pathology
}

\author{
Alyssa Caplan, Gabe Marx, Jonathan Elofson, Claire Lis, Lea Grinberg, Bruce Miller, and \\ Howard Rosen \\ Memory and Aging Center, University of California San Francisco, San Francisco, CA, USA
}

\begin{abstract}
Neurodegenerative diseases are caused by aggregation of specific proteins that catalyze a cascade of changes that ultimately lead to neurodegeneration. This concept guides current diagnostic approaches, as well as clinical trials, that focus on detecting or removing amyloid or tau from the brain. The semantic variant of primary progressive aphasia (svPPA), a clinical syndrome associated with frontotemporal lobar degeneration (FTLD) pathology, is usually associated with the molecular pathology TDP-C, but there are cases with TDP-B and Pick's disease. The existing literature on the clinical differentiation of these pathologies is limited. Here, we present a case study, in conjunction with a cross-sectional voxel-based morphometry (VBM), to elucidate the clinical and imaging features of a patient with svPPA due to Pick's disease.
\end{abstract}

\author{
Keywords \\ svPPA; semantic dementia; FTLD; Pick's disease; TDP-C
}

\section{Introduction}

In the study of neurodegenerative illness, the diagnosis begins with identification of the clinical syndrome based on a set of symptoms and findings, and secondarily involves an attempt to predict the microscopic pathology associated with the clinical syndrome. While the diagnosis of patients with FTD pathology is improving, predicting molecular pathology remains difficult. Thus, one of the goals of research in neurodegenerative disease is to identify clinical features that best predict microscopic pathology.

The clinical syndrome svPPA, is characterized by loss of semantic knowledge, severe anomia and single-word comprehension deficits (Hodges, Patterson, Oxbury, \& Funnell, 1992). When the neurodegeneration starts in the left temporal lobe the syndrome is usually language predominant, but when it begins in the right temporal lobe the syndrome is often dominated by dramatic behavioral changes including loss of empathy for others, rigidity, childish affect and difficulty with recognizing familiar faces (Edwards-Lee et al., 1997;

CONTACT Alyssa Caplan, Alyssa_Caplan@alumni.brown.edu.

Disclosure statement

No potential conflict of interest was reported by the authors. 
Seeley et al., 2005). In the UCSF brain bank over $80 \%$ of svPPA cases show, ubiquitinpositive (tau-negative, $a$-synuclein-negative) TDP-C aggregates. This is congruent with other pathological studies that find between 69 and $83 \%$ of cases with a clinical diagnosis of svPPA have TDP-C pathology at autopsy (Bonner, Ash, \& Grossman, 2010, Davies et al., 2005; Rohrer et al., 2011). Rarely, svPPA shows Alzheimer's disease pathology and typically these cases are associated with asymmetrical temporal lobe atrophy focused on the left hippocampus and superior temporal lobe, rather than the temporal pole and anterior and inferior temporal lobe as in classical svPPA caused by TDP-C pathology (Rohrer, Rossor, \& Warren, 2012). In some cases the pathology is not TDP-C or AD, but rather tau, usually Pick's pathology. The tau pathology of Pick's disease, is characterized by the presence of Pick bodies and 3R-tau-positive circumscribed neuronal cytoplasmic inclusions. Currently little is known about how to differentiate svPPA due to Pick's disease from that due to TDP-43 or AD. Here we present a case of svPPA with Pick's pathology, which could be potentially useful in helping physicians distinguish between pathologies.

\section{Methods and data}

\section{Clinical data collection}

This subject was referred to the UCSF Memory and Aging Center with a clinical diagnosis of frontotemporal dementia (FTD) and enrolled in a large research study. As part of this study, he underwent a neurological exam with a neurologist and a neuropsychological assessment with a trained neuropsychologist. T1-weighted MP-RAGE images were acquired on a 1.5-T Siemens Magnetom VISION system.

An autopsy was performed, and the brain was procured after death. Immunostaining for hyperphosphorylated tau (CP-13 antibody), $\beta$-amyloid 4G8, TDP-43, and a-synuclein was conducted.

\section{Case discussion}

Mr. G was a right-handed gentleman who had minor learning disabilities in his early childhood, including some difficulties with reading, math, and music. He performed well scholastically despite this. He was first seen at the UCSF Memory and Aging Center at the age of 66, with language, behavioral and personality changes, and memory loss beginning 6 years prior.

At age 57, he had a well-established career in marketing, when he suddenly decided to quit for no apparent reason, claiming that he "just wanted to do a different job." After this career change quickly ended in failure he returned to marketing. He was fired 2 years later, at age 59, for an outburst directed at his boss, and he retired permanently after this incident.

At the age of 61 he became overly fixated on minor medical problems, making frequent visits to his primary care physician who referred him to a psychologist. Anxiety was diagnosed and he obtained a prescription for an antidepressant which did not alleviate his symptoms. Around this time, his spouse noticed worsening memory and progressive difficulty following conversations, movie plots, and jokes. He developed difficulty remembering names and recognizing faces of acquaintances, and had trouble remembering 
the relationships these people had to him. For example, he did not recognize his sister-in-law whom he saw regularly.

One year before his visit to the Memory and Aging Center, he was started on Donepezil by his primary care physician, which led to no significant improvement. Six months later, he was seen by a neurologist, who conducted a thorough evaluation and PET scan. He concluded that Mr. G had FTD and referred him to the UCSF Memory and Aging Center.

At the time of his visit (age 66), Mr. G was having difficulties with recent memory, and exhibited repetitive questioning. His speech output was diminished, and he had word-finding difficulties with middle- and low-frequency words such as "suitcase," "bottle," and "gel." His wife noted that he had minimal spontaneous speech unless using a few words to ask for something that he needed. Also, he was having difficulty following oral commands and his wife often had to repeat instructions or physically help him start with a task. He was no longer able to drive due to easy distractibility. He became poor at multitasking and was also unable to complete household repairs. His motor skills were relatively spared. At this time, he was taking paroxetine $20 \mathrm{mg}$ daily, atorvastatin $40 \mathrm{mg}$ daily, and aspirin $81 \mathrm{mg}$ daily.

On neurological examination, he was well groomed and alert. He had a flat affect and was uninterested in conversation. He had a mild left upper motor neuron facial droop. On motor testing, he had increased tone in the left upper extremity, but otherwise normal tone. His rapid alternating movements were slightly clumsier on the left side than the right. No signs of amyotrophy or Parkinsonism were noted.

Family history, was negative for dementia. All genetic screening was non-contributory.

A neuropsychological evaluation showed deficits in verbal memory, as well as difficulties with naming pictures and diminished verbal fluency. Visual memory was below expectations as well. On the Peabody picture vocabulary test he was only able to identify $8 / 16$ pictures based on the words describing them. On abstract reasoning tests, he was only able to name $1 / 3$ similarities and $0 / 3$ proverbs. Also of note, he had significant difficulty identifying facial affect (Table 1).

A 1.5T MRI (Figure 1) showed severe temporal pole atrophy ( $>$ L), severe bilateral atrophy in the anterior temporal cortex, particularly in the right middle and inferior temporal gyri as well as the right fusiform gyrus. Severe dorsomedial frontal and moderate dorsolateral frontal atrophy was also present. Severe atrophy was also present in the anterior cingulate gyrus and the bilateral insulae $(\mathrm{R}>\mathrm{L})$. There was moderate atrophy in the right amygdala, and moderate atrophy in the orbitofrontal cortex.

After this visit, Mr. G received a diagnosis of the right temporal variant of svPPA.

Two years after the first visit, a phone follow-up interview with his wife revealed that he had been moved to a care home, and required assistance in most activities of daily living. He showed a decline in almost all elements of the Clinical Dementia Rating scale. He continued to have obsessions, and his preference for sweets progressed. He became incontinent and died at age 71 of throat cancer. 


\section{Autopsy results}

Exact brain weight and procurement time were not recorded at autopsy. Gross examination showed severe frontotemporal and mesial-temporal atrophy with severe ventricular enlargement. The cortical ribbon was severely thinned. On microscopic examination, severe superficial vacuolation, neuronal loss and astrogliosis were observed in the frontotemporal area. Immunostaining for hyperphosphorylated tau (CP-13 antibody) detected Pick bodies throughout cortical and subcortical structures. In addition, a large number of thorny astrocytes were present in the frontotemporal areas. No AD type neurofibrillary tangles were observed. Immunostaining for $\beta$-amyloid 4G8 only detected moderate numbers of diffuse plaques in cortical areas. TDP-43 and $\alpha$-synuclein immunohistochemistry were negative. Immunohistochemical analysis showed unequivocal evidence of classical Pick's disease, with abundant tau pathology in most regions examined, especially those implicated by the clinical syndrome svPPA. The tau pathology of Pick's disease, as seen in this case, is dominated by Pick bodies (tau-positive circumscribed NCIs), neuropil threads, astroglial inclusions and dsytrophic astrocytes, with gray matter more affected than white matter.

\section{Imaging data}

As Mr. G was given a diagnosis of the right temporal variant svPPA and we conducted a voxel-based morphometry (VBM) analysis to better understand the relationship between $\mathrm{Mr}$. G's pattern of brain atrophy and the pattern of atrophy in right temporal svPPA cases with the more common TDP-C pathology. Patients were categorized as right temporal variant with TDP-C pathology based both on clinical diagnosis and autopsy results. These subjects all had pathologically proven TDP-C pathology at autopsy. 8 subjects were included in the right temporal TDP-C variant group. In addition to being compared to each other, the case and the TDP-C group were compared to 67 age-matched normal controls. All subjects were enrolled in studies at the UCSF Memory and Aging Center.

MRI data was segmented using Statistical Parametric Mapping (SPM) 12 (http:// www.fil.ion.ucl.ac.uk/spm/) software. A study-specific template was created using the DARTEL toolbox (Ashburner, 2007). Tissue maps were normalized to template space by applying concatenated warps from native to study-specific template space and study-specific to template space. The normalized maps were subsequently Jacobian modulated and smoothed at 10 FWHM.

To compare the degree and topology of atrophy, W-Score maps were created for each patient's processed gray matter image based on previously published methodology (La Joie et al., 2012). W-scores are analogous to Z-scores but they adjust for specified covariates through general linear model regression. In this study, we created a model based from our 67 healthy controls that adjusted for age, total intracranial volume, and gender.

To directly compare the pattern of atrophy of the TDP-C patients to that of Mr. Gs, we created a mean W-score map averaged across each TDP-C patients' individual $\mathrm{W}$-score maps. 


\section{VBM analysis results}

In this VBM analysis, we see that the right temporal TDP-C cases (Figure 2, panel 3) generally have the most gray matter involvement in temporal regions, with extension into the posterior/medial portions of the frontal region and part of caudate, somewhat asymmetrically. The single right temporal Pick's case has frontal involvement that extends more anteriorly and appears more symmetrical (Figure 2, panel 1). This is visible in the gray matter maps, but the differences are accentuated in the white matter maps (Figure 2, panel 2)

\section{Discussion}

Mr. G came presented with language, behavioral and personality changes, and was given a diagnosis of right temporal, svPPA. On autopsy, he showed Pick's disease. His diagnosis of right temporal variant svPPA was based on behavioral changes such as impulsivity followed by verbal comprehension impairment and semantic deficits in language. He demonstrated worsening memory, prosopagnosia, and difficulty understanding facial affect. These clinical symptoms are all classic features of the right temporal variant, (Edwards-Lee et al., 1997), and correspond to degeneration in the right temporal lobe with some involvement of the left temporal lobe. On MRI, he showed marked atrophy of the right temporal pole and anterior temporal lobe, characteristic of right temporal variant svPPA. Mr. G showed additional symptoms such as severe apathy, associated with degeneration medial frontal structures (Rosen et al., 2005), which was present in his MRI scan.

In our VBM results comparing atrophy in a group of right temporal TDP-C svPPA cases to controls, we found severe asymmetric, localized involvement of temporal structures, including temporal poles and anterior inferior temporal cortex, and less marked involvement of orbitofrontal cortex, insula and anterior cingulate, which is consistent with the literature (Rohrer 2011). In comparing our Pick's case to controls, we found more involvement of the prefrontal cortex, the anterior cingulate, and the right caudate, with some involvement of the temporal lobes and orbitofrontal cortex. Less information is available in the literature regarding the pattern of Pick's atrophy, although it can be strongly asymmetric (Rohrer 2011). According to the literature, the pattern of Pick's atrophy involves the prefrontal and orbitofrontal cortex, with some involvement of the temporal lobes, insula, and anterior cingulate, and sparing of parietal lobes (Rohrer 2011, Whitwell \& Josephs, 2012). We found significantly more white matter atrophy in the Pick's case as compared to the TDP-C cases. This is consistent with previous studies that have suggested significant gray and white matter pathology in tau cases (Forman et al., 2002) whereas TDP-C cases generally have greater gray matter atrophy (Geser et al., 2009).

Few studies have described differences in the clinical presentation of svPPA due to Pick's pathology versus the more commonly found TDP-C pathology. One study found acalculia to be a distinguishing feature of svPPA due to Pick's pathology (Rohrer 2011), however, Mr. G had no reported problems with calculations and made no calculation errors on neuropsychological testing. 


\section{Conclusion}

The finding of Pick's pathology in a case of right temporal variant svPPA is unusual, as this clinical diagnosis is strongly associated with TDP-43 type C pathology. Using a combination of subjects from a literature search, it was found that out of 77 cases of svPPA, 57 (74\%) cases were due to TDP-C pathology, and 9 (11\%) had Pick's (Davies, 2005, Rohrer 2011, \& Bonner, 2010). Another study selected subjects with a right temporal atrophy pattern, and separated these cases by a clinical diagnosis of bvFTD and svPPA. At autopsy, all 8 subjects with right temporal variant bvFTD showed tau pathology, and all 3 with svPPA had TDP-C pathology (Josephs et al., 2009).

In this paper we aimed to use a case discussion to elucidate ways to differentiate between TDP-C and Pick's pathology in a svPPA case. Although this study has limited applicability due to its nature as a case study, we were able to highlight regional gray and white matter differences on MRI. The right temporal TDP-C group had the most gray matter involvement in temporal regions, with extension into the posterior/medial portions of the frontal region and part of caudate, somewhat asymmetrically. The single Pick's case has frontal involvement that extends more anteriorly and appears more symmetrical and had significantly more white matter atrophy. In the future, larger pathological studies are needed to be able to clearly differentiate pathologies during life as it is this idea that guides current diagnostic approaches, as well as clinical trials, that focus on treating single, specific proteins.

\section{Acknowledgments}

\section{Funding}

This publication was made possible by grant [Grant Number P01 AG019724] from NIH National Institute on Aging [Grant Number R01 AG032306] from NIH National Institute of Neurological Disorders and Stroke, and a grant from the Tau Consortium.

\section{References}

Ashburner J. A fast diffeomorphic image registration algorithm. NeuroImage. 2007; 38:95-113. DOI: 10.1016/j.neuroimage.2007.07.007 [PubMed: 17761438]

Bonner MF, Ash S, Grossman M. The new classification of primary progressive aphasia into semantic, logopenic, or nonfluent/agrammatic variants. Current Neurology and Neuroscience Reports. 2010; 10:484-490. DOI: 10.1007/s11910-010-0140-4 [PubMed: 20809401]

Bonner MF, Ash S, Grossman M. The new classification of primary progressive aphasia into semantic, logopenic, or nonfluent/agrammatic variants. Current Neurology And Neuroscience Reports. 2010; 10:238-490. DOI: 10.1007/s11910-010-0140-4

Davies RR, Hodges JR, Kril J, Patterson K, Halliday G, Xuereb J. The pathological basis of semantic dementia. Brain : A Journal of Neurology. 2005; 128:1984-1995. DOI: 10.1093/brain/awh582 [PubMed: 16000337]

Davies RR, Hodges JR, Kril J, Patterson K, Halliday G, Xuereb J. The pathological basis of semantic dementia. Brain : A Journal Of Neurology. 2005; 128:1984-1995. DOI: 10.1093/brain/awh582 [PubMed: 16000337]

Edwards-Lee T, Miller BL, Benson DF, Cummings JL, Russell GL, Boone K, Mena I. The temporal variant of frontotemporal dementia. Brain : A Journal of Neurology. 1997; 120:1027-1040. DOI: 10.1093/brain/120.6.1027 [PubMed: 9217686] 
Forman MS, Zhukareva V, Bergeron C, Chin SS, Grossman M, Clark C, Lee VM. Trojanowski JQ Signature tau neuropathology in gray and white matter of corticobasal degeneration. The American Journal of Pathology. 2002 Jun.160:2045-2053. DOI: 10.1016/S0002-9440(10)61154-6 [PubMed: 12057909]

Geser F, Martinez-Lage M, Robinson J, Uryu K, Neumann M, Brandmeir NJ, Trojanowski JQ. Clinical and pathological continuum of multisystem TDP-43 proteinopathies. Archives of Neurology. 2009 Feb.66:180-189. DOI: 10.1001/archneurol.2008.558 [PubMed: 19204154]

Hodges JR, Patterson K, Oxbury S, Funnell F. Semantic dementia. Progressive fluent aphasia with temporal lobe atrophy. Brain : A Journal of Neurology. 1992; 115:1783-1806. DOI: 10.1093/brain/ 115.6.1783 [PubMed: 1486461]

Josephs KA, Whitwell JI, Knopman DS, Boeve BF, Vemuri P, Senjem ML, Jack CR. Two distinct subtypes or right temporal variant frontotemporal dementia. Neurology. 2009; 73:1443-1450. DOI: 10.1212/WNL.0b013e3181bf9945 [PubMed: 19884571]

La Joie R, Perrotin A, Barre L, Hommet C, Mezenge F, Ibazizene M, Chetelat G. Region-specific hierarchy between atrophy, hypometabolism, and beta-amyloid (Abeta) load in Alzheimer's disease dementia. The Journal of Neuroscience : the Official Journal of the Society for Neuroscience. 2012; 32:16265-16273. DOI: 10.1523/JNEUROSCI.2170-12.2012 [PubMed: 23152610]

Rohrer JD, Lashley T, Schott JM, Warren JE, Mead S, Isaacs AM, Warren JD. Clinical and neuroanatomical signatures of tissue pathology in frontotemporal lobar degeneration. Brain : A Journal of Neurology. 2011; 134:2565-2581. DOI: 10.1093/brain/awr198 [PubMed: 21908872]

Rohrer JD, Lashley T, Schott JM, Warren JE, Mead S, Isaacs AM, Warren JD. Clinical and neuroanatomical signatures of tissue pathology in frontotemporal lobar degeneration. Brain : A Journal Of Neurology. 2011; 134:2565-2581. DOI: 10.1093/brain/awr198 [PubMed: 21908872]

Rohrer JD, Rossor MN, Warren JD. Alzheimer's pathology in primary progressive aphasia. Neurobiology of Aging. 2012; 33:744-752. DOI: 10.1016/j.neurobiolaging.2010.05.020 [PubMed: 20580129]

Rosen HJ, Allison SC, Schauer GF, Gorno-Tempini ML, Weiner MW, Miller BL. Neuroanatomical correlates of behavioral disorders in dementia. Brain : A Journal of Neurology. 2005; 128:26122625. DOI: 10.1093/brain/awh628 [PubMed: 16195246]

Seeley WW, Bauer AM, Miller BL, Gorno-Tempini ML, Kramer JH, Weiner M, Rosen HJ. The natural history of temporal variant frontotemporal dementia. Neurology. 2005; 64:1384-1390. DOI: 10.1212/01.WNL.0000158425.46019.5C [PubMed: 15851728]

Whitwell JL, Josephs KA. Recent advances in the imaging of frontotemporal dementia. Current Neurology and Neuroscience Reports. 2012; 12:715-723. DOI: 10.1007/s11910-012-0317-0 [PubMed: 23015371] 


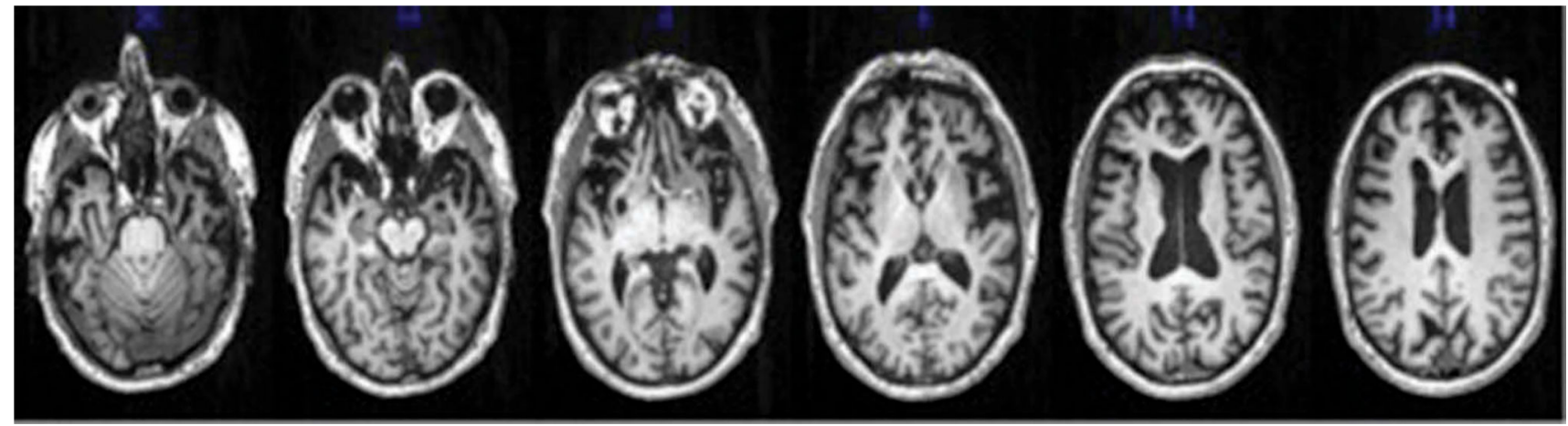

Figure 1.

1.5T MRI of case.

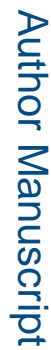

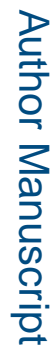

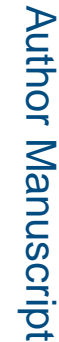



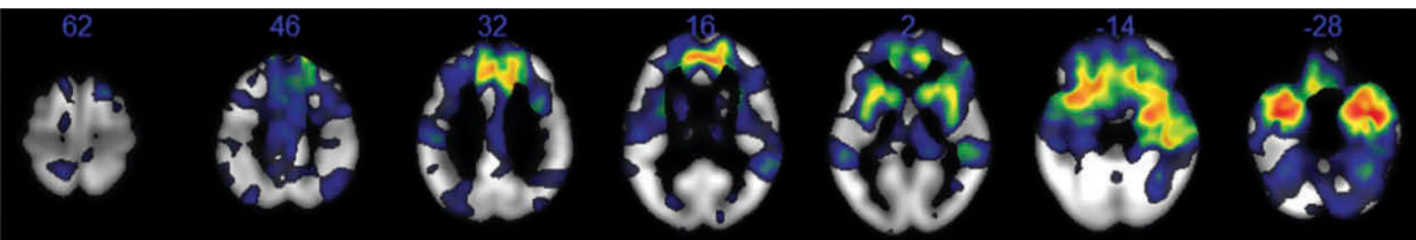

If

46

62

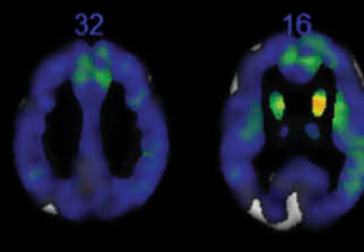

$6 . \lambda$

ㄴำ
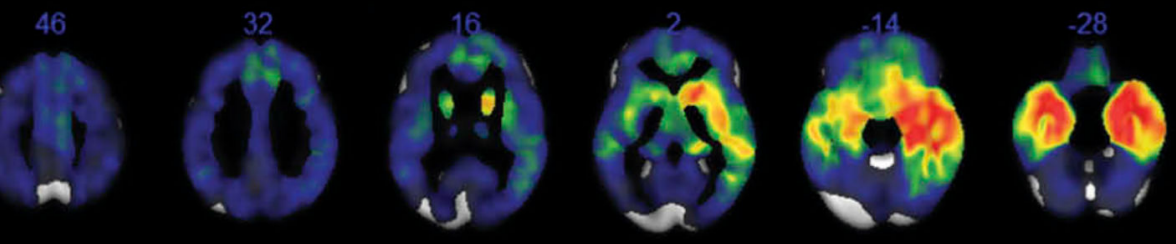

$-44$

62

46

32

16

$-14$

$-28$

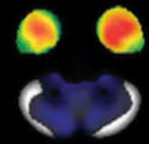

i.
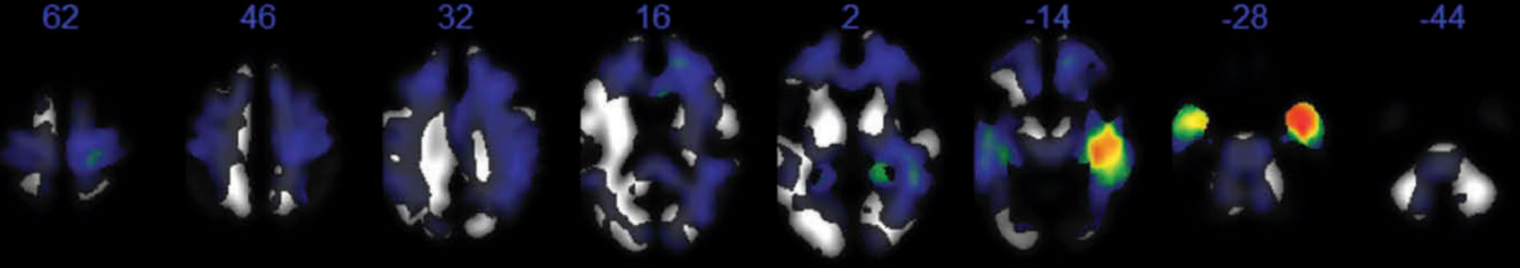

$-44$

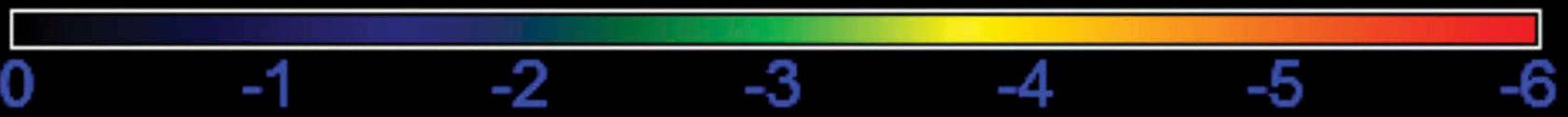

Figure 2.

W-Score maps.

Panel 1: Case: W-Score Map Gray

Panel 2. Case: W-Score Map White

Panel 3. TDP: W-Score Map Gray

Panel 4. TDP: W-Score Map White 


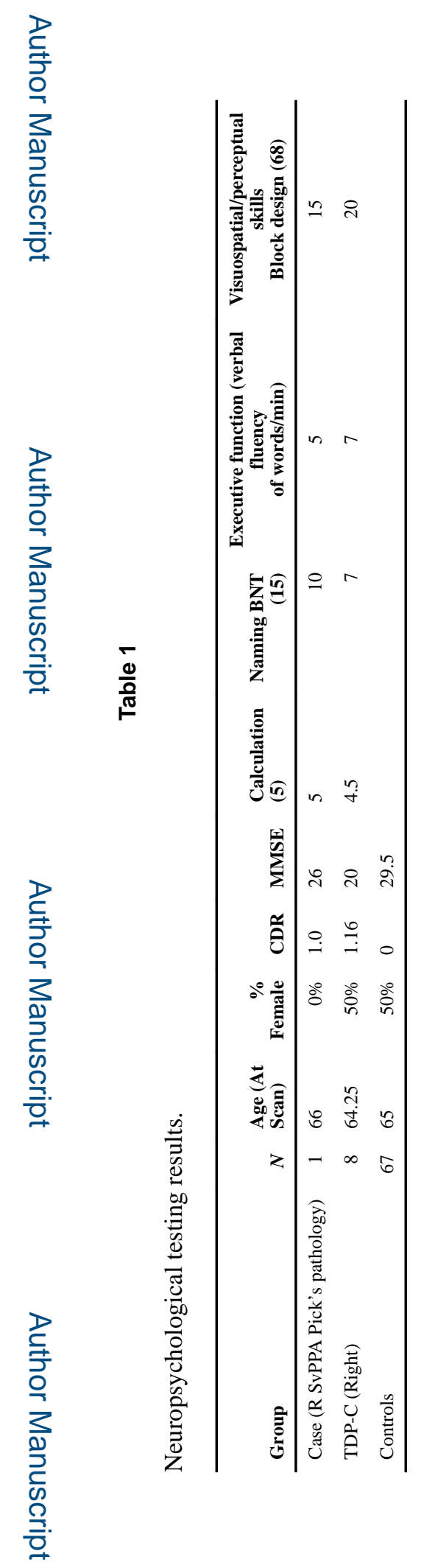

Neurocase. Author manuscript; available in PMC 2019 April 01. 